\title{
Montgomery and shared decision- making: implications for good psychiatric practice
}

\author{
Gwen Adshead, David Crepaz-Keay, Mayura Deshpande, K.W.M (Bill) Fulford and \\ veryan Richards
}

\section{Summary}

The 2015 Supreme Court judgment in Montgomery v Lanarkshire Health Board [2015] UKSC 11 established that consent to medical treatment requires shared decision-making based on dialogue between the clinician and patient. In this editorial, we examine what Montgomery means for standards of good psychiatric practice, and argue that it represents an opportunity for delivering best practice in psychiatric care.

\section{Declaration of interest}

None.

\section{Keywords}

Ethics; consent and capacity; psychiatry and law.

\section{Copyright and usage}

(c) The Royal College of Psychiatrists 2018.
Gwen Adshead (pictured) is a forensic psychiatrist with a long-standing interest in mental health ethics. David Crepaz-Keay is a psychiatric patient who has promoted the importance of survivor voices in mental health policy, practice and future alternatives. Mayura Deshpande is a consultant in adolescent forensic psychiatry, with an interest in the philosophy and ethics of psychiatry and application of policy in practice. K.W.M (Bill) Fulford is a psychiatrist and philosopher working on values in healthcare. He is the Founder Director of Oxford's Centre for Values-based Practice. Veryan Richards is a patient representative at the Royal College of Psychiatrists. As a lay participant, she contributes toward shaping the policy narrative and improving patient experience in several areas of mental healthcare.
In 2015, the UK Supreme Court gave judgment in a case establishing a new legal standard for consent to medical treatment. ${ }^{1}$ In this editorial, we discuss the implications of Montgomery $v$ Lanarkshire Health Board [2015] UKSC 11 for good practice and training in psychiatry, beginning with an overview of the judgment.

\section{The facts of the case}

Mrs Montgomery was under the care of Dr McClellan, a consultant obstetrician. During her pregnancy, she developed insulin-dependent diabetes, which is known to carry a relatively rare risk of shoulder dystocia in a baby delivered vaginally. Mrs Montgomery asked about potential risks during labour, but Dr McClelland decided against warning Mrs Montgomery of the risks of vaginal delivery because she considered the risks of the alternative (i.e. a caesarean section) to be greater than those of shoulder dystocia. She was also motivated in this by her experience that, if given the option, women in Mrs Montgomery's position tended to opt for caesarean section.

Sadly, the labour was delayed by shoulder dystocia and the baby was born with severe disabilities arising from prolonged hypoxia. Mrs Montgomery sued for negligence, arguing that Dr McClelland had breached her duty of care by not advising her of the risks of vaginal delivery and of the option of a caesarean section. Two lower courts found against Mrs Montgomery on the grounds that Dr McClelland had acted in accordance with a standard accepted as proper by a responsible body of medical opinion (the test of negligence established in the case of Bolam $v$ Friern Hospital
Management Committee [1957] 1 WLR 582, 587, ${ }^{2}$ widely called the Bolam test).

The Supreme Court, however, relying heavily on the General Medical Council's (GMC) standards of Good Medical Practice and related guidance on consent, ${ }^{3}$ reversed the lower courts' decision and found in favour of Mrs Montgomery. The Bolam test, the Court argued, although still appropriate for technical aspects of clinical care (diagnosis and treatment), was no longer appropriate for decisions about treatment. Therefore, a new test, reflecting contemporary standards of good practice, was required.

\section{Implications for consent}

Despite considerable debate about the precise implications of Montgomery, there is general agreement that, post-Montgomery, consent requires a process of shared decision-making based on dialogue between clinician and patient about the material risks and the benefits of the available options (including the option of no treatment).

The dialogue required by Montgomery retains the perspective of the clinician as well as adding the importance of the perspective of the patient. This is made clear in the Montgomery two-limbed test of a material risk. This is defined as a risk that is considered material, either from the perspective of a reasonable person in the patient's position (first limb of the test), or from that of the particular patient concerned (second limb of the test) (Montgomery at 87 per Lord Kerr and Lord Reed). The legal arguments supporting this test recognise that the patient and clinician may see the risks and benefits of treatment options differently in terms of their values, i.e. what matters most or is important to them. The dialogue enables both perspectives to be explored.

\section{Implications for good psychiatric practice}

The Montgomery judgment presents both challenges and opportunities for clinicians in day-to-day mental health practice. Some obvious initial challenges include issues that are known to be ethically challenging in psychiatry, such as prejudice and discrimination in relation to some diagnostic/patient groups; the influence of thirdparty interests, such as employers and service providers; and 
concerns that the interests of public safety often weigh more heavily than patient welfare.

Space does not permit full rehearsal of all the challenges cited above; insofar as they are indirectly affected by Montgomery judgment. However, there is a specific risk of discrimination arising at the interface between Montgomery and other legislation on consent, notably the Mental Health Act 2007 (MHA) and the Mental Capacity Act 2005 (MCA). Montgomery assumes 'soundness of mind' (at 50 per Lord Kerr and Lord Reed) and, in another passage, 'capacity' (at 115 per Lady Hale). A possible reading of Montgomery might be that psychiatry is, by virtue of these passages, exempted from its conclusions. This would be a highly discriminatory reading, however, because it would assume that all psychiatric patients are either of unsound mind or lack capacity.

The implications of these passages of Montgomery will no doubt be explored in forthcoming reviews of the MCA and MHA, but the key point for now is that neither passage provides any general exemption for psychiatry from the model of consent in Montgomery. Both the MCA and MHA have very strictly defined and delimited scopes of application. Montgomery indicates by implication that neither should be used merely to pre-empt what is important to the patient concerned (at 91 per Lord Kerr and Lord Reed). This is consistent with the fact that even where the MCA or MHA is applicable, both Acts (like Montgomery) require attention to the particular patient's values (respectively by way of the best interests principle of the MCA and the guiding principles for the MHA). Herring et al ${ }^{4}$ argue that Montgomery, far from exempting psychiatry, has in effect extended the principle of supported decision-making at the heart of the MCA to everyone.

So how does the Montgomery model of consent work in practice in mental health services? Consider, by way of example, consent to treatment with medication that has a high risk of side-effects, such as second-generation antipsychotics. These medications carry a recognised risk of metabolic side-effects, including weight gain leading to diabetes. Patients are aware of these risks and express concern about them, ${ }^{5}$ and there are indications that they may underestimate the risks. ${ }^{6}$

The parallels with Montgomery are striking. Just like Dr McClelland, psychiatrists may be reluctant to disclose all the risks associated with antipsychotic medication because of the potential benefits of the treatment and the likelihood that the patient will not accept the medication if they hear all the risks. In both cases, clinicians believe that non-disclosure is in their patients' best interests. However, in both cases, the risks may well be considered material by the particular patients concerned (second limb of the Montgomery materiality test), and it is likely that a reasonable person in the patient's position would consider the risks to be material (first limb of the Montgomery materiality test).

For example, clinicians may not discuss side-effects of psychoactive medications that they consider minor, such as transient gastrointestinal upsets, dry mouth and increased photosensitivity. The physician may feel that the benefits of treatment entirely outweigh such minor and transient effects. However, such effects may be highly significant and distasteful to patients, so much so that they may decide to refuse the medication. Further, different patients may weigh side-effects in highly individual ways, such that the clinician feels that it is better to not discuss the more minor side-effects for this reason.

Pre-Montgomery, the Bolam test was available in defending the practice of non-disclosure because psychiatrists could argue that they were acting in accordance with a standard accepted as proper by a responsible body of medical opinion. Post-Montgomery, by contrast, this argument is no longer defensible because Bolam is no longer applicable. According to the Supreme Court judgment, a patient's consent to antipsychotic medication requires the psychiatrist concerned to have engaged in dialogue with them about the risks and benefits not just of the proffered antipsychotic, but of the options available (including the no treatment option), to the point that a choice can be made that, as Montgomery puts it, 'take(s) into account her (the patient's) own values' (at 115 per Lady Hale).

\section{Challenges for good psychiatric practice}

The Montgomery ruling presents several other challenges. One generic challenge is a concern about increased risks of litigation by patients, especially in a risk-adverse National Health Service culture, where psychiatrists fear being blamed for any adverse consequence associated with a jointly made decision. Anticipating such concerns, Montgomery points out that in a genuinely shared process of decision-making the patient takes a degree of responsibility alongside the clinician. In theory, this should result in reduced risk of litigation (at 93 per Lord Kerr and Lord Reed) when decision-making is shared, but only time will tell.

A second, perhaps even greater, generic challenge arising from the requirements of Montgomery involves the increased time required for shared decision-making. For example, Lee ${ }^{7}$ has queried whether it is feasible to expect doctors to know enough about patients to predict what they would want to know. Montgomery argues against such concerns, noting that similar approaches to consent have been successfully implemented in other administrations, notably Australia (at 70-72 per Lord Kerr and Lord Reed).

A challenge from Montgomery specific to psychiatry is the often emotionally charged nature of psychiatric consultations. Research into language and communication about risk suggests that emotions may affect communication outside the consciousness of the speaker, especially in high-risk or otherwise charged situations. ${ }^{8}$ Both doctors and patients may be affected by these emotions, which in mental health settings, can include fear of coercion or assault and feelings of powerlessness. ${ }^{9}$ Obvious examples of highly charged discussions about consent include difficult discussions regarding leave in suicidal patients, food intake with patients with anorexia nervosa and therapeutic work with people who self-harm or refuse treatment.

\section{Opportunities for good psychiatric practice}

The challenges to implementing Montgomery are arguably derived from and inherent in established GMC standards (at 77 and 78 per Lord Kerr and Lord Reed). Some have argued in light of its reliance on GMC guidance (and related legal precedent) that when it comes to its implications for practice, Montgomery changes little. ${ }^{10}$ Our view is to the contrary, namely that in raising the status of shared decision-making from guidance to legal requirement, Montgomery changes everything. This is because with Montgomery the requirement for shared decision-making is no longer optional but essential to valid consent.

Shared decision-making as we have indicated is challenging: it requires resources of time and a willingness on the part of the psychiatrist, equipped with good communication skills, to engage in a partnership approach and dialogue with the patient. But these requirements, based as they are on the GMC's model of consent, are not (merely) legal requirements but the requirements of good practice. This is why Montgomery is an opportunity for good psychiatric practice. So long as shared decision-making was optional there was no real incentive to provide the resources to support this. Now that it has to be done the resources have to be found. Again, a full discussion of the resource implications of Montgomery is beyond the scope of this editorial.

In mandating shared decision-making, Montgomery actually strengthens the importance of the psychiatrist's expertise on the 
technical aspects of the decision. Patients are increasingly informed about treatment options through the ready availability of web-based information. However, the variable quality of such information makes it even more important that the clinician brings to shared decision-making secure evidence-based knowledge about the options available. In the case of antipsychotic medication (our example above), this means being up-to-date with the latest information about the risks and benefits of the wide range of available options, including alternatives to medication, combined approaches and the option of no treatment.

National Institute for Health and Care Excellence (NICE) guidelines and locally agreed protocols may be helpful in this regard as they are helpful to the evidence base of shared decisionmaking. What is perhaps less well-recognised is that they are helpful also to the values-base of shared decision-making. NICE guidelines emphasise that, 'when exercising their judgment, professionals are expected to take this guideline fully into account, alongside the individual needs, preferences and values of their patients or service users.' Over 70 of the guidelines published by NICE refer to the need for professionals to take account of the individual preferences and values of patients or service users; therefore like Montgomery, NICE guidelines add consideration of values alongside evidence to professional expertise.

\section{Next steps: resources and the role of the Professional Practice and Ethics Committee}

The Royal College of Psychiatrists' newly configured Professional Practice and Ethics Committee (PPEC) is tasked with revising and updating Good Psychiatric Practice, i.e. the standards that the GMC use to judge psychiatric conduct. A first and perhaps key role for the PPEC in updating Good Psychiatric Practice will be to set out standards for consent consistent with Montgomery (and its underpinning GMC standards); standards that recognise both the challenges and opportunities set out above. These standards will need to address the interface issues noted earlier with the MCA and MHA. A further role for the PPEC will be to seek evidence of what, if any, additional resources (such as consultation time) are required for implementing Montgomery in psychiatry and to advise the College accordingly. In working on revised guidance, PPEC will liaise with other groups in the College, such as those that represent patients and carers, and those working on the legal interface with the MHA and MCA.

Another area of collaboration will be the current review of the curriculum. Montgomery makes clear the essential role of communications skills in shared decision-making, and how learning such skills is no longer optional. Training methods for communicating about values have been developed for specialties such as surgery and could be adapted for psychiatry. ${ }^{11}$ College policy developments, such as the report of the Values-based Child and Adolescent Mental Health System Commission ${ }^{12}$ and the report Core Values for Psychiatrists, ${ }^{13}$ will also provide an important resource in setting out the required standards and reviewing the curriculum and training.

\section{Conclusions}

We have argued that the 2015 Supreme Court Montgomery judgment provides an opportunity for psychiatry to put shared decision-making based on evidence and values at the heart of good psychiatric practice. This would be consistent with GMC standards of good medical practice. It would be consistent also with person-centred approaches to clinical decision-making in psychiatry aimed at recovery and development of self-management skills. This is why implementing
Montgomery will be at the forefront of the PPEC's forthcoming review of Good Psychiatric Practice. In this, the PPEC will work in partnership with other groups within the College and draw on initiatives in values-based practice in other medical specialties.

The shared decision-making model of consent mandated by Montgomery reflects a wider move towards more collaborative working relationships between professionals and patients in healthcare. Co-production, as it is widely called, has often proved easier to name than to enact. In developing its work in this area the PPEC will seek to embody the principles of co-production by ensuring that its revised guidance on good psychiatric practice is a product of genuine dialogue between clinicians and patients aimed at coming to a shared view of what is important in this most challenging area of clinical care.

Gwen Adshead, MBBS, FRCPsych, MA, Consultant Forensic Psychiatrist and Psychotherapist, Hampshire Pathfinder Service, Southern Health Foundation Trust and Consultant Forensic Psychiatrist and Psychotherapist, HMP Bronzefield, Camden \& North West London Foundation Trust, UK; David Crepaz-Keay, DProf, Head of Empowerment and Social Inclusion, Mental Health Foundation; Fellow, Institute of Mental Health and Partner, The Collaborating Centre for Values-based Practice, St Catherine's College, Oxford, UK; Mayura Deshpande, MBBS, MRCPsych, Consultant Forensic Psychiatrist and Associate Medical Director, Southern Health NHS Foundation Trust and Chair, Professional Practice \& Ethics Committee, Royal College of Psychiatrists, London and Clinical Lead, National Network of Adolescent Medium Secure Services, NHS England, UK; K.W.M (Bill) Fulford, DPhil, FRCP, FRCPsych, Fellow, St Catherine's College, Oxford and Member, Philosophy Faculty, University of Oxford and Emeritus Professor, Philosophy and Mental Health, University of Warwick and Founder Editor and Chair of the Advisory Board, Philosophy, Psychiatry, \& Psychology Journal and Director, The Collaborating Centre for Values-based Practice, St Catherine's College, Oxford, UK; Veryan Richards, BSC Hons, Individual Partner of the Collaborating Centre for Values-based Practice in Health and Social Care, St Catherine's College, Oxford, UK

Correspondence: Gwen Adshead, Hampshire Pathfinder Service, Southern Health NHS Foundation Trust, Ravenswood House, Mayles Lane, Fareham, Hampshire P017 5NA, UK. Email: g.adshead@nhs.net

First received 19 Jun 2018, accepted 12 Aug 2018

\section{References}

1 Montgomery v Lanarkshire Health Board [2015] UKSC 11.

2 Bolam v Friern Hospital Management Committee [1957] 1 WLR 582, 587.

3 General Medical Council. Consent: Patients and Doctors Making Decisions Together. General Medical Council, 2008.

4 Herring J, Fulford KWM, Dunn D, Handa A. Elbow room for best practice? Montgomery, patients' values, and balanced decision-making in personcentred care. Med Law Rev 2017; 25(4): 582-603.

5 Karthik M, Kulhara P, Chakrabarti S. Attitude towards second-generation antipsychotics among patients with schizophrenia and their relatives. Hum Psychopharmacol Clin Exp 2013; 28: 457-65.

6 Gao K, Fang F, Wang Z, Calabrese JR. Subjective $v$. objective weight gain during acute treatment with second-generation antipsychotics in schizophrenia and bipolar disorder. J Clin Psychopharmacol 2016; 36(6): 637-42.

7 Lee A. 'Bolam' to 'Montgomery' is result of evolutionary change of medical practice towards 'patient-centred care. Postgrad Med J 2017; 93(1095): 46-50.

8 Slovic $\mathrm{P}$, Peters E, Finucane ML, MacGregor DG. Affect, risk, and decision making. Health Psychol 2005; 24(Suppl 4): S35-40.

9 Katsakou C, Bowers L, Amos T, Morriss R, Rose D, Wykes T, et al. Coercion and treatment satisfaction among involuntary patients. Psychiatr Serv 2010; 61(3): 286-92.

10 Heywood R. R.I.P. Sidaway: patient-oriented disclosure-a standard worth waiting for? Montgomery v Lanarkshire Health Board [2015] UKSC 11. Med Law Rev 2015; 23: 455-66.

11 Person-centred Training and Curriculum Scoping Group. Person-Centred Care: Implications for Training in Psychiatry. Royal College of Psychiatrists, 2018.

12 Values-based Child and Adolescent Mental Health System Commission. What Really Matters in Children and Young People's Mental Health. Royal College of Psychiatrists and on behalf of Young Minds and the Children and Young People's Mental Health Commission, Values-Based Child and Adolescent Mental Health System Coalition, 2016.

13 Richards V, Lloyd K. Core Values for Psychiatrists: Report CR204. Royal College of Psychiatrists, 2017. 